\title{
Global Perspectives on Adolescent Health and Economic Strengthening: Lessons from Sub-Saharan Africa
}

\author{
Fred M. Ssewamala ${ }^{1,2} \cdot$ Vilma Ilic $^{2}$
}

Published online: 28 April 2015

(C) Springer International Publishing 2015

Over one third of the global population is under the age of 19, and $90 \%$ of these youth live in developing countries. Nearly half of developing country youth live on less than \$2 a day, and poverty exacerbates threats to wellbeing. In sub-Saharan Africa, the interactive effects of youth poverty and disease are particularly severe. Decades of economic crisis, the AIDS pandemic, and civil conflict have left millions of African youth orphaned or otherwise vulnerable while simultaneously weakening family support systems. Fifteen million youth in sub-Saharan Africa have lost at least one parent to HIV/AIDS.

These youth disproportionately miss out on education and are more likely to engage in risk-taking behavior such as unprotected sex and substance abuse. An equally significant number are affected by wars and civil conflict. If unaddressed, the mutually reinforcing crises of youth poverty and disease threaten fragile development gains, suggesting a devastating downward spiral in human development over the next generation. Recognizing the urgency of the crises, researchers, practitioners, academics, and programmers came together to exchange ideas, showcase evidence, share lessons learned from field studies, and offer suggestions for future research at the Global Perspectives on Adolescent Health and Economic

Fred M. Ssewamala

fs2114@columbia.edu

Vilma Ilic

vpi2101@columbia.edu

1 Columbia University School of Social Work, 1255 Amsterdam Avenue, New York, NY 10027, USA

2 International Center for Child Health and Asset Development, Columbia University School of Social Work, 1255 Amsterdam Avenue, Office 1122, New York, NY 10027, USA
Strengthening Conference: Lessons from Sub-Saharan Africa, the first ever conference on adolescent health and economic strengthening that provided a forum for exchange, dissemination, and development of innovative economic strengthening research in sub-Saharan Africa over the past decade. Conference participants discussed the future of economic strengthening programs in low-resource communities, especially communities affected by poverty and disease, including HIV/ AIDS, and children in emergency situations. In this special issue, we are honored to present papers from this conference.

This special issue showcases empirical research and review articles revealing the latest findings and discoveries in the burgeoning fields of child protection programs and economic strengthening interventions for children, adolescents, and their caregiving families in regions experiencing adversities, such as poverty, disease, conflict, and humanitarian crises.

In Protecting Assets for the Most Vulnerable: The Potential for Asset-Based Interventions, Njeri Kagotho provides an overview of the legal framework in Kenya and Uganda intended to protect vulnerable and socially excluded populations, particularly women, children, and people living with HIV. In practice, however, these laws fail to safeguard the populations they target. When these groups are susceptible to disenfranchisement, programs are needed to fill gaps where laws fail. Among the successful interventions are those that have incorporated components of asset building by connecting disenfranchised populations to formal financial institutions. Kagotho stresses the versatility of asset-building programs given their reach, scope, and documented success.

Leyla Karimli, Fred Ssewamala, Torsten Neilands, and Mary McKay examine the savings behavior of poor families and orphaned children in Uganda using data from a cluster randomized controlled trial in Matched Child Savings Accounts in Low Resource Communities: Who Saves? Their analyses demonstrate that orphaned children who participated 
in the matched savings program saved enough to invest in post-primary education, and children were more likely to save and make deposits knowing that their caregivers saved as well. However, orphaned children who were being cared for by a living parent utilized their savings accounts more often, compared to orphaned children who were cared for by other relatives. These, and additional findings of their study, contribute to an understanding of saving behavior among children and caregivers living in poverty, and add knowledge to the feasibility of social welfare policies focused on asset building.

Li Zou and colleagues remind us of the dearth of qualitative research on savings patterns and behavior, in particular low savings rates, among young people in sub-Saharan Africa, and attempt to reduce this gap with their results presented in a paper titled Facilitators and Obstacles in Youth Saving: Perspective from Ghana and Kenya. Their paper provides qualitative evidence from youth in Ghana and Kenya, two countries participating in the YouthSave initiative. The YouthSave initiative is a 5 -year project that aims to investigate the potential of savings accounts as a tool for youth development and financial inclusion in four developing countries: Colombia, Ghana, Kenya, and Nepal. Using a multiple case study approach across Ghana and Kenya that include in-depth interviews with youth participants, parents or caregivers, and school stakeholders, the research team aimed to better understand what youth consider to both facilitate and hinder savings and encourage participation in a savings program. Their findings are consistent with other research noting that institutional mechanisms, including financial institutions, are important in facilitating youth savings. Concerning obstacles, Zou and colleagues indicate that youth noted limited employment and competition for funds as barriers to saving, as well as a lack of guidance on saving and financial management. These results can be informative for policymakers and financial institutions promoting youth savings programs.

Keeping the focus on vulnerable children, specifically those orphaned due to AIDS, a paper titled, Relationship Between Family Economic Resources, Psychosocial Wellbeing, and Educational Preferences of AIDS-orphaned Children in Southern Uganda: Baseline Findings, examines the extent to which economic resources and orphanhood status affect the educational, psychosocial, and economic preferences of AIDS-orphaned children and adolescents. Ssewamala and colleagues demonstrate that AIDS-orphaned children have high educational expectations and aspirations; however, single orphans (defined as children who have lost one biological parent) have higher educational aspirations compared to double orphans who have lost both parents. This could be because double orphans most likely have less psychosocial and economic support compared to single orphans. Conversely, the authors found that both single and double orphans reported similar levels of psychosocial functioning. Finally, the analyses in the Ssewamala and colleagues paper also revealed an association between economic preferences, educational aspirations, and psychosocial wellbeing. These baseline findings are notable because they call attention to that fact that despite their vulnerabilities, AIDS-orphaned children have hopes, dreams, and aspirations for the future and should be supported to maintain these positive ambitions throughout primary school and as they transition to secondary education. Supportive measures may include combined economic strengthening, empowerment, and psychosocial support programs.

In the fifth paper of this special issue, Rising School Enrollment and Declining HIV and Pregnancy Risk among Adolescents in Rakai District, Uganda, 1994-2013, Dr. John Santelli and colleagues used a mixed methods approach, combining quantitative and qualitative data to underscore the role of education in healthy child and adolescent outcomes in the short term as well as across the lifespan, confirming the need for investing in economic strengthening and asset-building programs stipulated for education. The quantitative and qualitative data came from the Rakai Community Cohort Study in Uganda involving adolescents ages 15-19 years from 1994 to 2013. The analyses reveal that rising socioeconomic status contributed to rising school enrollment. In qualitative interviews, youth reported a lack of money, the death of parents, and becoming pregnant as primary reasons for school dropout. The authors note that increases in school enrollment were associated with delays in social transitions such as marriage and initiation of sexual intercourse, and with declines in risk for HIV and pregnancy among adolescent women. Increasing access to schooling and efforts to retain adolescents in school can provide important improvements in adolescent sexual and reproductive health, including reduction of HIV infection. With such conclusive results reinforcing the central role of schools in increasing health and wellbeing among adolescents, it is imperative for children and adolescents to remain in school. In the context of sub-Saharan Africa, continued efforts aimed at economically empowering orphaned and vulnerable children and adolescents are urgently needed.

Last but not least, in Barriers to Utilization of HIV Care Services among Adolescents and Young Adults in Rakai, Uganda: The Role of Economic Strengthening, Dr. Gertrude Nakigozi and her team of medical doctors at Rakai Health Sciences Program present their qualitative findings from their decades-long ongoing cohort studies with HIV-positive adolescents and young adults in southern Uganda. Their previous work indicates that young people ages 15-24 in Uganda are the least likely to access and utilize HIV care services compared to all other HIV-positive age groups. Utilization of HIV care services includes antiretroviral therapy (ART), and optimal adherence to ART is a requisite for its efficacy. Suboptimal adherence results in drug resistance, rendering ART ineffective. Overall, Nakigozi and colleagues' findings 
indicate that economic barriers are highly prevalent as obstacles to utilization of care, suggesting a need for economic strengthening for adolescents and young adults living with HIV in Uganda.

Decisions that young people make today influence not only their future as individuals but societies are influenced as well, particularly societies in which young people make up the overwhelming majority of the population, as is the case in sub-Saharan Africa, the region of focus for this special issue. As we read the articles in this special issue, it is evident that researchers continue to test and investigate programs and policies that positively impact young people's health and development and protect them from risks.
We hope this special issue will contribute to the ongoing discussion and knowledge base on adolescent health and economic strengthening. And we hope that the Global Perspectives on Adolescent Health and Economic Strengthening Conference will become an annual or biannual event. We thank the Child Protection in Crisis Learning Network, United States Agency for International Development, McSilver Institute for Poverty Policy and Research, International Center for Child Health and Asset Development, Columbia University School of Social Work, and the University of Chicago School of Social Service Administration, and its Center for Health Administration Studies for their generous support and for making the inaugural conference possible. 